\title{
Flow directions of low-energy ions in and around the diamagnetic cavity of comet 67P
}

\author{
Sofia Bergman ${ }^{(0)}, 1,2 \star$ Gabriella Stenberg Wieser ${ }^{(0,},{ }^{1}$ Martin Wieser, ${ }^{1}$ Hans Nilsson ${ }^{\circledR}$, ${ }^{1}$ Erik Vigren ${ }^{(0)},{ }^{3}$ \\ Arnaud Beth ${ }^{\circledR},{ }^{2}$ Kei Masunaga ${ }^{4}$ and Anders Eriksson ${ }^{\circledR 3}$ \\ ${ }^{1}$ Swedish Institute of Space Physics, Box 812, SE-981 28 Kiruna, Sweden \\ ${ }^{2}$ Department of Physics, Umeå University, SE-901 87 Umeå, Sweden \\ ${ }^{3}$ Swedish Institute of Space Physics, Box 537, SE-751 21 Uppsala, Sweden \\ ${ }^{4}$ Institute of Space and Astronautical Science, Japan Aerospace Exploration Agency, Yoshinodai 3-1-1, Chuo-ku, Sagamihara, Kanagawa 252-5210, Japan
}

Accepted 2021 August 26. Received 2021 August 16; in original form 2021 June 15

\begin{abstract}
The flow direction of low-energy ions around comet 67P/Churyumov-Gerasimenko has previously been difficult to constrain due to the influence of the spacecraft potential. The Ion Composition Analyzer of the Rosetta Plasma Consortium (RPC-ICA) on Rosetta measured the distribution function of positive ions with energies down to just a few eV/q throughout the escort phase of the mission. Unfortunately, the substantial negative spacecraft potential distorted the directional information of the low-energy data. In this work, we present the flow directions of low-energy ions around comet 67P, corrected for the spacecraft potential using Particle-In-Cell simulation results. We focus on the region in and around the diamagnetic cavity, where low-energy ions are especially important for the dynamics. We separate between slightly accelerated 'burst' features and a more constant 'band' of low-energy ions visible in the data. The 'bursts' are flowing radially outwards from the nucleus with an antisunward component while the 'band' is predominantly streaming back towards the comet. This provides evidence of counter-streaming ions, which has implications for the overall expansion velocity of the ions. The backstreaming ions are present also at times when the diamagnetic cavity was not detected, indicating that the process accelerating the ions back towards the comet is not connected to the cavity boundary.
\end{abstract}

Key words: plasmas - methods: data analysis - methods: numerical-comets: individual: 67P/Churyumov-Gerasimenko.

\section{INTRODUCTION}

Low-energy ions play important roles in cometary environments. When the neutral particles in the coma get ionized, primarily through photoionization but also through electron-impact ionization and charge exchange with the solar wind (Galand et al. 2016; Simon Wedlund et al. 2017), the newly created ions are initially cold and moving with the same velocity as the neutral gas $\left(0.5-1 \mathrm{~km} \mathrm{~s}^{-1}\right.$; Gulkis et al. 2015). The subsequent motion of the newborn lowenergy ions is determined by electrodynamics. A radial ambipolar electric field, created due to the charge separation arising from warm electrons in a steep density gradient, accelerates the ions outwards from the comet nucleus (Vigren \& Eriksson 2017; Berčič et al. 2018; Nilsson et al. 2020). The different gyro radii of ions and electrons furthermore give rise to a tailward polarization electric field (Nilsson et al. 2018), resulting in a flow direction of new born ions radially outwards from the nucleus with a clear antisunward component (Berčič et al. 2018).

When the solar wind encounters the plasma cloud around the comet, the cometary particles are 'picked up' and accelerated along the convective electric field of the solar wind, forcing the solar wind

^E-mail: sofia.bergman@irf.se to get decelerated and/or deflected (e.g. Behar et al. 2016, 2018). This process is known as mass loading (e.g. Szegö et al. 2000). As a result, the interplanetary magnetic field (IMF) is draped around the comet nucleus (Alfvén 1957). Since comets do not have an intrinsic magnetic field (Auster et al. 2015), any magnetic field in the vicinity of the comet must have been transported from the solar wind or be the result of currents induced by the solar wind-coma interaction. At both comet 1P/Halley (Neubauer et al. 1986) and comet 67P/ChuryumovGerasimenko (hereafter 67P; Goetz et al. 2016a,b) a magnetic field free region, a diamagnetic cavity, was observed in the innermost part of the comet magnetosphere. For comet 1P/Halley, this region was shown to be collisionally dominated with ion velocities similar to the velocity of the neutrals (e.g. Schwenn et al. 1987). It was suggested that the ion-neutral drag force is balancing the magnetic pressure at the cavity boundary (Ip \& Axford 1987; Cravens 1989). For comet $67 \mathrm{P}$, however, the ion velocity has been found to be significantly higher than the neutral velocity (Bergman et al. 2021; Odelstad et al. 2018; Vigren et al. 2017), indicating that the ions are not as strongly coupled to the neutrals at less active comets. The processes responsible for the formation and maintenance of the diamagnetic cavity at less active comets is hence uncertain, and information about the flow direction of the low-energy ions in this region is necessary for a deepened understanding. 
(a)

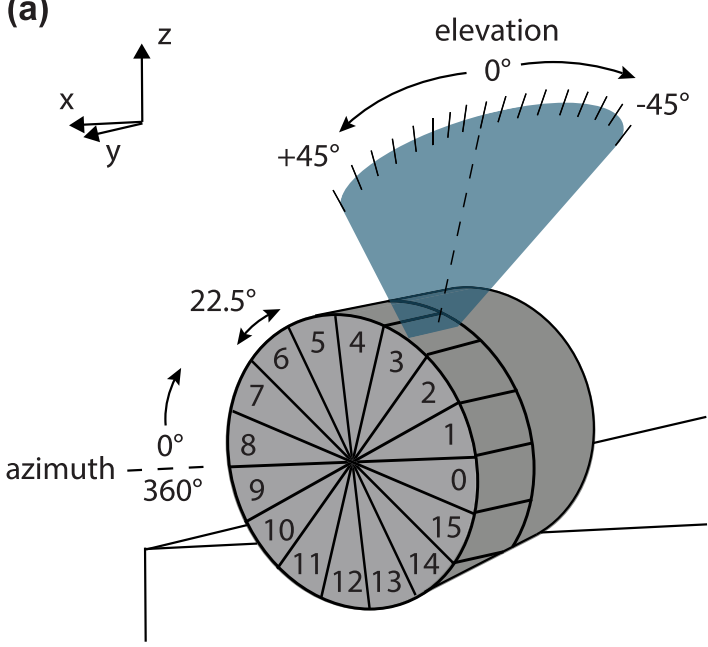

(b)

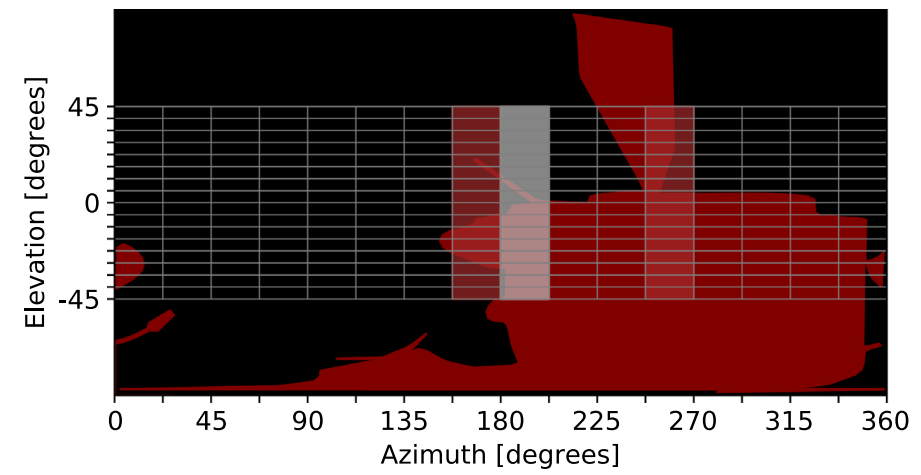

Sector

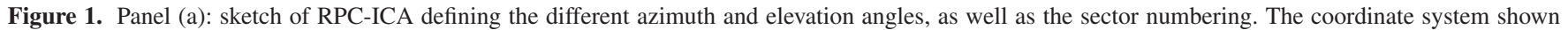

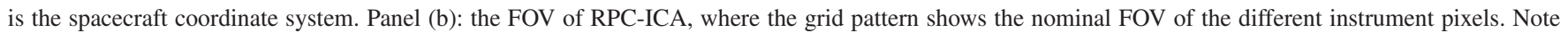

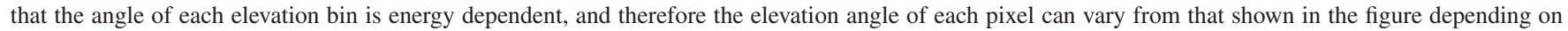

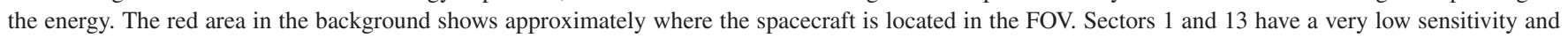

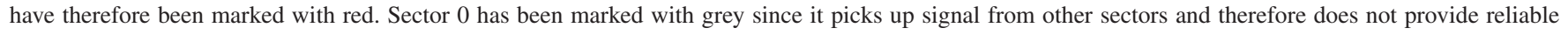
information about the directions.

Unfortunately, the analysis of low-energy ions is complicated due to the influence of the spacecraft potential. The Rosetta spacecraft (Glassmeier et al. 2007a), studying comet 67P between $2014 \mathrm{Au}-$ gust and 2016 September, was charged to a negative potential of typically -10 to $-20 \mathrm{~V}$ throughout the mission (Odelstad et al. 2017; Johansson et al. 2020). This complicated the interpretation of measurements made by plasma instruments on board. The Ion Composition Analyzer of the Rosetta Plasma Consortium (RPCICA; Nilsson et al. 2007) was an ion mass spectrometer on-board Rosetta, capable of measuring ions with energies down to just a few eV/q with an angular resolution of $22.5 \times 5.6 \mathrm{deg}^{2}$. RPCICA was hence able to study the dynamics of the low-energy ions in the environment. However, the negative spacecraft potential accelerated the ions towards the spacecraft prior to detection, which for low-energy ions significantly affected the energy spectrum and directional information of the data. Bergman et al. (2020b) used the Spacecraft Plasma Interaction Software (SPIS; Thiébault et al. 2013) to model the influence on the ion trajectories and the resulting distortion of the effective field of view (FOV) of RPC-ICA for different ion energies. The results showed that the distortion can be considered insignificant when the ion energy (in units of eV) is more than twice the absolute value of the spacecraft potential (in units of volts). The distortion at lower energies is highly geometry dependent, with a different distortion for each individual pixel of the instrument. Bergman et al. (2020a) furthermore showed that the FOV distortion is dependent on the Debye length of the surrounding plasma, where some pixels are more sensitive to a varying Debye length than others. The distortion pattern is hence very complicated, and needs to be modelled taking the geometry of the spacecraft and the properties of the surrounding plasma into account.

The simulation results presented by Bergman et al. (2020a,b) make it possible to constrain the flow directions of low-energy ions around comet $67 \mathrm{P}$ for the very first time. In this study, we present the first results from this correction, focusing on the flow directions in the region inside and in the vicinity of the diamagnetic cavity.

\section{INSTRUMENT DESCRIPTION}

\subsection{The Ion Composition Analyzer}

The Ion Composition Analyzer of the Rosetta Plasma Consortium (RPC-ICA; Nilsson et al. 2007) is a mass resolving ion spectrometer sampling the three dimensional distribution function of positive ions. The energy is analysed in an electrostatic analyser, covering an energy range of a few $\mathrm{eV} / \mathrm{q}$ to $40 \mathrm{keV} / \mathrm{q}$ with a nominal resolution of $\mathrm{d} E / E=0.07$. The energy range is covered by sweeping over 96 energy steps.

\subsubsection{Field of view}

RPC-ICA has a total nominal FOV of $360 \times 90 \mathrm{deg}^{2}$. The FOV of one individual instrument pixel is $22.5 \times 5.6 \mathrm{deg}^{2}$, where 22.5 is the angular resolution in the azimuth direction and 5.6 is the angular resolution in the elevation direction. In azimuth the angular resolution is achieved by physically dividing the cylindrically symmetric instrument into 16 sectors, while in elevation different angles are achieved through electrostatic deflection. This deflection system consists of two plates put to different potentials, and by varying the potential difference between the plates, ions with different elevation angles (with respect to the aperture plane) can enter the instrument. The elevation range from $-45^{\circ}$ to $+45^{\circ}$ is scanned in 16 steps (yielding a resolution of 5.6). In Fig. 1(a), a sketch of RPC-ICA is shown with a definition of the different azimuth and elevation angles. The sector numbering is also shown.

In Fig. 1(b), the complete nominal FOV of the instrument is shown. The grid cells correspond to the nominal FOV of each individual instrument pixel. The red area in the background shows the approximate location of the spacecraft within the FOV. It is clear that the spacecraft body and solar panels obstruct a significant part of the FOV. Mainly, it is the negative elevation angles of sectors 9-15 and 0 that suffer from shadowing effects. The sectors marked with red in Fig. 1(b) (sectors 1 and 13) have a very low sensitivity and consequently the instrument is basically blind in these directions. 
Sector 0 has been marked with grey in Fig. 1(b) since it picks up signal from the other sectors. RPC-ICA uses position sensing on its micro channel plate to determine the mass bin and the azimuthal sector for each detected particle. With a certain probability the sector number can at times not be uniquely determined due to ambiguous inputs to the instrument electronics. In this case, a fixed sector 0 is assigned to the detection, independent from the actual arrival direction of the particle. From a data analysis point of view, this results in sector 0 containing a small fraction of counts from all other sectors. Therefore, this sector does not yield reliable directional information and has been excluded from this study.

\subsubsection{Time resolution}

The nominal time resolution of RPC-ICA is $192 \mathrm{~s}$. However, a mode with higher resolution was implemented upon arrival at the comet in order to capture the fast variations of the dynamic ion environment (Stenberg Wieser et al. 2017). This mode has a resolution of $4 \mathrm{~s}$, which was achieved by confining the measurement to one elevation bin and only sweeping over energies up to $82 \mathrm{eV} / \mathrm{q}$. The confinement to one elevation bin results in a nearly two dimensional FOV of $360 \times$ $5.6 \mathrm{deg}^{2}$. The elevation angle of the bin used is estimated to be close to $0^{\circ}$ for sufficiently high sensor temperatures ( $>13.5 \mathrm{C}$ ), but may increase with a few degrees for lower temperatures. In energy, the high-time-resolution mode gives a better coverage of the low-energy population due to denser sampling. In this study, we exclusively use data obtained with the high-resolution mode.

\subsection{The Langmuir probes}

The Langmuir probe instrument on-board Rosetta (RPC-LAP; Eriksson et al. 2007) can obtain measurements of several plasma parameters such as effective ion flow speed, plasma density and electron temperature, which is done by measuring the currents flowing to the probe. It can also obtain estimates of the spacecraft potential, which are used in this study. For details regarding spacecraft potential determinations using RPC-LAP the reader is referred to Odelstad et al. (2017) or Johansson et al. (2021), but the basic principle is to estimate the potential either from the photoelectron knee observed in a sweep $\left(V_{\mathrm{ph}}\right)$ or from the probe floating potential $\left(V_{\mathrm{f}}\right) . V_{\mathrm{ph}}$ provides better estimates when the potential is close to zero or positive, but otherwise $V_{\mathrm{f}}$ is less sensitive to noise. An empirical model was proposed by Johansson et al. (2021), mapping $V_{\mathrm{f}}$ to the equivalent $V_{\text {ph }}$ values:

$U_{\mathrm{s} / \mathrm{c}}=V_{\mathrm{f}}+5.5 \exp \left(\frac{V_{\mathrm{f}}}{8.0}\right)$,

where all numbers are in volts. In this study, we use $V_{\mathrm{f}}$ estimates of the spacecraft potential, corrected using equation (1). We furthermore assume that RPC-LAP picks up 85 per cent of the full spacecraft potential. This fraction was shown by Odelstad et al. (2017) to vary between 70 and 100 per cent. However, no method has as yet been found to reliably determine this fraction for individual intervals.

\section{METHOD}

\subsection{Data selection}

RPC-ICA was operated in the high-time-resolution mode during 88 cavity observations, ranging in time from 2015 May to 2016
February. For a few observations, the sensor temperature was too low for a satisfactory sampling of the ion distribution (see Section 3.2 for details about the sensor temperature's influence on the data). These data are removed from the study, together with a few other observations with inconvenient binning of the data. In total, 75 cavity observations are used for the analysis. A complete list of these observations can be found in Appendix A.

In Fig. 2, the energy-time spectrum for each sector of RPC-ICA is shown for one cavity observation. Between the dashed vertical lines Rosetta was located within the diamagnetic cavity. This example illustrates the typical appearance of the RPC-ICA data within the cavity (see also fig. 1 in Bergman et al. 2021). The plasma in and around this region typically appears as a constant 'band' of lowenergy ions mixed up with sporadic bursts of slightly accelerated ions. To distinguish between these two features throughout this paper, we will refer to them as the 'band' and the 'bursts', respectively. Bergman et al. (2021) showed that the energy distribution of the ions in the band is characterized by a Maxwellian shape with a bulk speed of 5-10 $\mathrm{km} \mathrm{s}^{-1}$ and a temperature of $0.7-1.6 \mathrm{eV}$. Typically, the spacecraft potential is constant in regions where only the band is present. The bursts, on the other hand, are often accompanied by a fast varying spacecraft potential and a plasma density increase. Masunaga et al. (2019) analysed the flow direction of the accelerated part of these features $(>40 \mathrm{eV})$ and suggested that they are accelerated by the tailward polarization electric field (Nilsson et al. 2018) from upstream of the comet. A large part of these bursts have steepened shapes (Type 4, as defined by Stenberg Wieser et al. (2017)). These steepened plasma enhancements, appearing both inside and outside of the cavity, were analysed by Hajra et al. (2018) using data from the Mutual Impedance Probe (RPC-MIP; Trotignon et al. 2007). They suggested that the enhancements observed inside of the cavity are transmitted from outside of this region, but whether this means a mass transfer across the boundary or a compression by an impermeable boundary is still uncertain. Outside of the cavity, the steepened features observed in the particle data are usually accompanied by equivalent features in the magnetic field data, which were analysed by Ostaszewski et al. (2021) and identified as fast magnetosonic waves. The propagation of these waves was shown to be perpendicular to the background magnetic field. Note though that we do not make a separation between different burst features in this study. Type 2 and Type 5 features as defined by Stenberg Wieser et al. (2017) are occasionally present as well, but have not been separated from the steepened features.

The data in Fig. 2 have not been corrected for the spacecraft potential. However, already in this uncorrected shape we see indications of different flow directions for the ions in the bursts compared to the band. The band is mainly present in sectors 14 and 15 (and 0 , but as previously mentioned, sector 0 picks up signal from other sectors and is hence not reliable), while the bursts mainly appear in sectors 4-8 (and 2, but the sensitivity of sector 2 is slightly higher than the other sectors and this signal should therefore be analysed with care). This motivates a separation of the data comprising the band and the bursts. The directional information will be analysed separately for each feature.

Time periods only containing the band can be easily distinguished in energy-time spectra, and we manually pick out time periods only containing these data. To separate the bursts from the band, a simple filtering of the data is needed, since the band is continuously present. For each cavity observation, we calculate a mean intensity of the band (if such intervals are present) for each energy step (corrected for the spacecraft potential) and sector, and subtract it from the whole interval. 

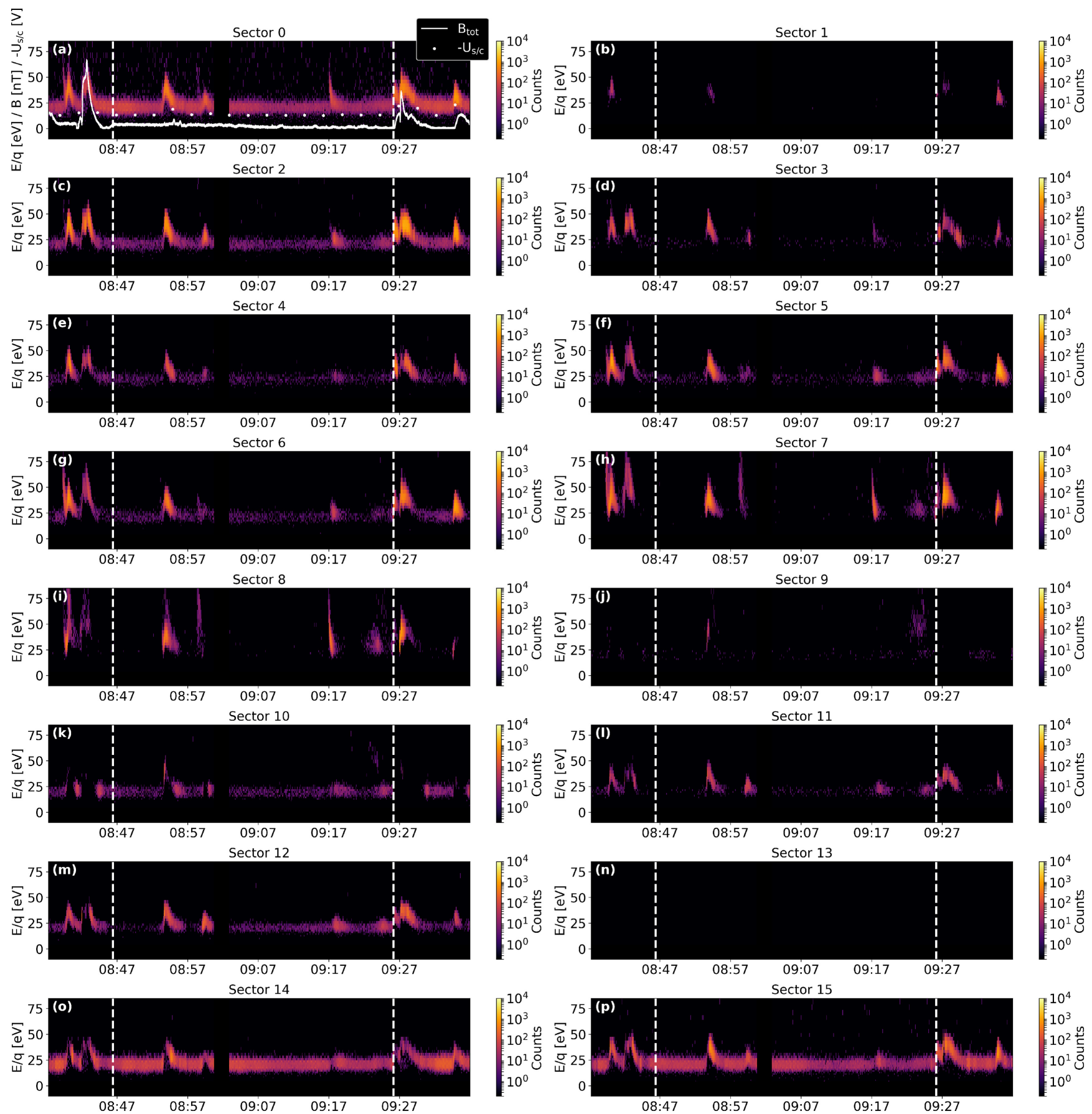

Figure 2. Energy-time spectra obtained with RPC-ICA on 2015 November 20. The different panels show the spectrum obtained with each azimuthal sector. Between the dashed vertical lines, Rosetta was located within the diamagnetic cavity. The band is predominantly present in sectors 14 and 15 (panels o and p), while the bursts appear in sectors 4-8 (panels e-i). Sector 0 picks up signal from the other sectors and sector 2 is slightly more sensitive than the other sectors. These spectra should therefore be observed with care. Sectors 1 and 13 have a very low sensitivity. In panel (a), the total magnetic field strength as measured by the magnetometer (RPC-MAG; Glassmeier et al. 2007b) on Rosetta is overplotted as a white solid line. Spacecraft potential estimates from RPC-LAP is shown as white dots in the same panel. The RPC-ICA data have not been corrected for the spacecraft potential. Sectors $4-5$ are looking towards the comet.

\subsection{Correction of the energy scale}

The energy scale of RPC-ICA suffers from offsets, caused by mainly two parameters: low sensor temperatures and the spacecraft potential. Low sensor temperatures $(<13.5 \mathrm{C})$ cause internal high voltage drifts, shifting the energy scale towards higher energies. As a consequence, parts of the ion distribution can end up below the energy range covered by the instrument at that time. These data have been removed from the study, as mentioned in Section 3.1. However, often the shift is small enough for the whole distribution to still be covered and the energy scale can instead be corrected.

The spacecraft potential additionally causes an energy shift of the ions according to $-q U_{\mathrm{s} / \mathrm{c}}$, where $q$ is the ion charge and $U_{\mathrm{s} / \mathrm{c}}$ the spacecraft potential. This shift can be corrected for using spacecraft potential estimates from RPC-LAP. However, the resolution of the 
RPC-LAP data is often not sufficient to cover the fast variations of the potential, especially observed in the bursts (see Fig. 2a). We therefore use an alternative method to correct for the spacecraft potential that also takes care of the drifts caused by the sensor temperature: the lower cut-off observed in the RPC-ICA spectra.

In the diamagnetic cavity, the local ionization creates ions with energies down to nearly $0 \mathrm{eV}$. When these are accelerated by the spacecraft potential they are detected by RPC-ICA at an energy of $-q U_{\mathrm{s} / \mathrm{c}}$, which creates a cut-off in the energy spectrum. From this cutoff, we can get a high resolution estimate of the spacecraft potential. If the sensor temperature is low, this causes an additional shift of the energy scale. However, assuming that the energies of the ions in the cut-off were originally zero $\mathrm{eV}$, we can correct the energy scale for both sensor temperature and spacecraft potential offsets by shifting the energy scale so that the cut-off appears at this energy. For the band intervals, we set the cut-off to the first energy bin where the number of counts $\geq 5$. Furthermore, the following three bins need to contain a monotonically increasing amount of counts or at least one bin with nine or more counts (same criteria as set by Odelstad et al. 2017, which was shown to produce a cut-off estimate not markedly sensitive to noise). For the bursts, we increase the required number of counts for the first bin to $\geq 10$. The signal is stronger in the bursts and the noise level makes it difficult to properly capture the full shape of the spikes if the level is not increased. To further reduce the influence of noise, we use a moving average boxcar filter with a window size of 3 for the bursts. For the band, the spacecraft potential is relatively constant and we reduce the influence of noise by calculating one average offset for the whole interval.

\subsection{Correction of flow directions}

In order to analyse the flow directions, the directional information of the data has to be corrected for the spacecraft potential. We use the simulation results from Bergman et al. (2020b) for this correction.

\subsubsection{Simulations}

Detailed information about the simulations used for the correction has already been presented by Bergman et al. (2020b), and the interested reader is referred to that source. However, a brief summary is given here.

SPIS is a Particle-In-Cell (PIC) solver modelling spacecraft plasma interactions. Given a plasma environment, illumination conditions and spacecraft characteristics, the current balance between the spacecraft surface and the plasma is solved for and the resulting spacecraft potential and potential field around the spacecraft determined. The influence on particle measurements can be investigated through particle tracing. Bergman et al. (2020b) used the particle tracing tool of SPIS to determine the distortion of the effective FOV of each pixel of RPC-ICA for different ion energies. The plasma model used in these simulations represent the environment of the comet close to perihelion, with a density of $1000 \mathrm{~cm}^{-3}$ (Henri et al. 2017), an electron temperature of $8 \mathrm{eV}$ (Eriksson et al. 2017), an ion temperature of $0.5 \mathrm{eV}$ (Galand et al. 2016), and a radial bulk velocity of the ions of $4 \mathrm{~km} \mathrm{~s}^{-1}$ (Odelstad et al. 2018). This results in a spacecraft potential of $-21 \mathrm{~V}$, which is a low but reasonable value, actually observed around perihelion.

One example of the simulation results is shown in Fig. 3. The dashed square represents the nominal FOV of the studied pixel, while the coloured area corresponds to the effective (distorted) FOV at this particular energy range and spacecraft potential $(5-10 \mathrm{eV}$ and $-21 \mathrm{~V}$,

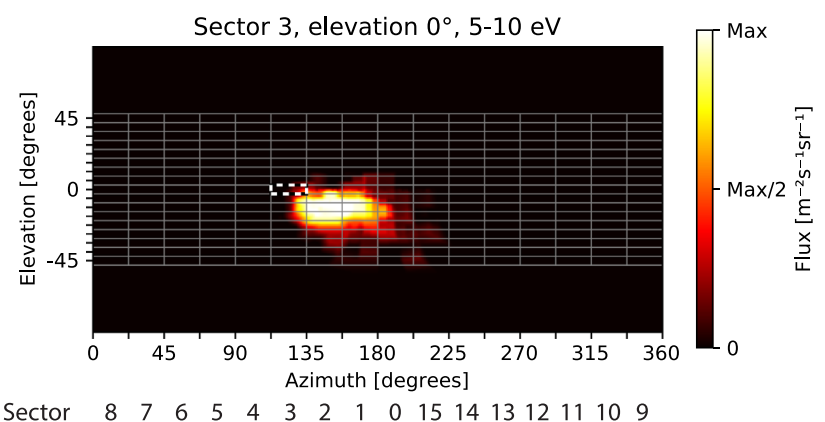

Figure 3. Simulation result showing the distortion of one instrument pixel of PRC-ICA when the ion energy before the ions are accelerated by the spacecraft potential is $5-10 \mathrm{eV}$ and the spacecraft potential is $-21 \mathrm{~V}$. The pixel shown has a nominal FOV as shown by the dashed square (sector 3 , elevation angle $0^{\circ}$ ). The colour scale shows the flux of particles at the external boundary reaching the pixel from different directions. To improve the statistics, five simulations as presented in Bergman et al. (2020b) have been combined to produce the figure. Bergman et al. (2020b) simulated 17 elevation bins instead of 16 , and therefore the grid pattern differs from that shown in Fig. 1(b) (see Bergman et al. 2020b for more details).

respectively). The colour scale represents flux of particles at the external boundary (edge of the simulation volume, corresponding to undisturbed plasma) reaching the pixel from different directions. When the energy is increased, the effective FOV approaches the nominal.

It was shown by Bergman et al. (2020a) that statistical variations of the SPIS solver introduces uncertainties to the particle tracing results. To reduce these uncertainties and improve the statistics, we run additional simulations with identical setup as Bergman et al. (2020b) (but with different random sampling of initial particle positions and velocities) and combine these results. That is, we produce five flux maps similar to the one shown in Fig. 3 and average them. This yields results with smaller statistical uncertainty than those presented in the previous study.

\subsubsection{Assumptions}

The simulations done by Bergman et al. (2020b) have limitations. Only one spacecraft potential and only one plasma environment (resulting in the same Debye length for all simulations) were modelled. Of course, both the spacecraft potential and the Debye length vary between the different cavity observations analysed in this study. Furthermore, the cold electron population reported by e.g. Eriksson et al. (2017), Gilet et al. (2020), and Wattieaux et al. (2020), which is important for an accurate modelling of the Debye length, has not been included in the simulations by Bergman et al. (2020b).

To study different spacecraft potentials, we use a simple interpolation, assuming a linear relation between spacecraft potential and ion energy. For example, if the observed spacecraft potential for a measurement is half of the simulated potential $(-10.5 \mathrm{~V})$ and we want to correct energies of 5-10 eV, we double the energy interval in the simulations and use the results for $10-20 \mathrm{eV}$. The relation between spacecraft potential and ion energy was shown by Bergman et al. (2020a) not to be perfectly linear. However, this mainly affected the size of the FOV, while the effect on the FOV centre direction was not significant. We therefore expect this assumption to have a negligible effect on the final result.

Different Debye lengths, however, were shown by the same study to also affect the position of the centre of the FOV. A smaller Debye length generally results in a more severe distortion of the FOV. 

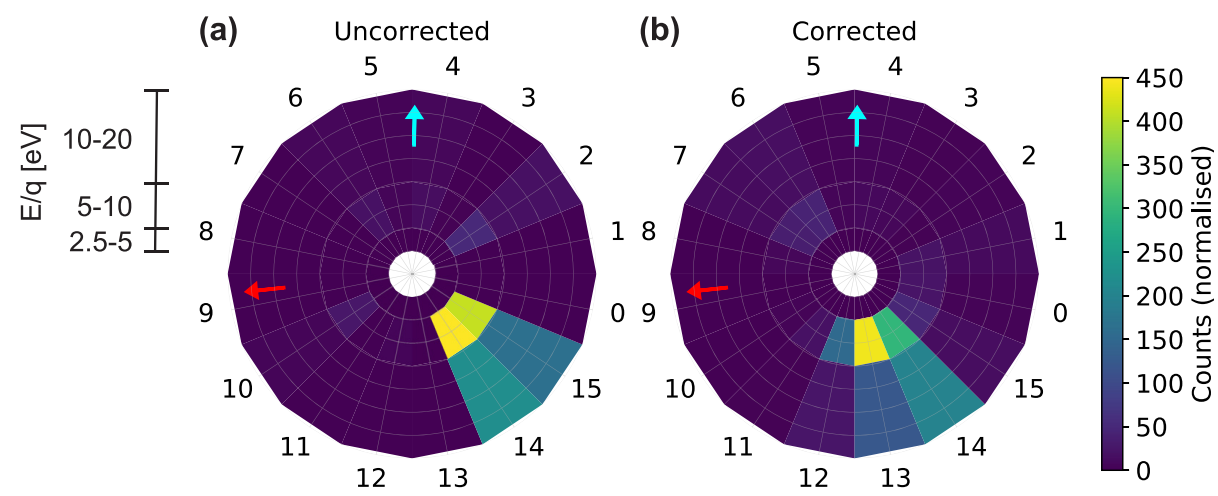

Figure 4. Example data from 2015 November 20, from 08:46:35 to 08:52:51 (panel a) before and (panel b) after correcting the directional information. The numbers on the azimuthal axis correspond to the sectors of RPC-ICA and the radial axis corresponds to energy. The colour scale corresponds to counts detected from different directions, normalized by the observation time for each energy interval. For example, an ion moving in the antisunward direction would result in a signal in sector 9. The blue and red arrows show the average comet and Sun directions, respectively, for this particular interval. The spacecraft potential is $-13 \mathrm{~V}$, and hence the lowest energy interval $(2.5-5 \mathrm{eV})$ has not been included.

However, a different Debye length mainly causes a shift of the FOV in the elevation direction (see fig. 9 in Bergman et al. 2020a). Since the high-time-resolution measurements are made in nearly $2 \mathrm{D}$, we are only doing a correction in the azimuthal direction in this study. We therefore assume that the error introduced by the assumption of a constant Debye length $(0.66 \mathrm{~m}$, as yielded by the modelled environment) is small.

However, an increase in the size of the effective FOV naturally leads to a larger uncertainty in the correction. The distorted FOV for one instrument pixel can be large, and the detected signal is likely not broad enough to cover the complete FOV. However, when correcting the directions we assume that this is the case, which causes an artificial broadening of the signal after correction. This broadening becomes larger for shorter Debye lengths and less negative spacecraft potentials.

In the study by Bergman et al. (2020b), the potential field around the spacecraft was modelled assuming a bulk flow of the ions in the radial direction $(-z$-direction in spacecraft coordinates, for the typical nadir pointing of Rosetta). However, in cases where the results presented in this study indicate a flow in a different direction, this also results in a slightly different potential field around the spacecraft (since the location of the wake changes). This would affect the particle tracing results, and we analyse the resulting uncertainty in Section 4.1.

Finally, assumptions about the elevation angle used by RPC-ICA in the high-time-resolution mode have to be made. As already mentioned, the elevation angle at high sensor temperatures is expected to be close to the aperture plane, while the angle increases with some degrees at lower temperatures. However, when the elevation angle is varied, the effective FOV is mainly shifted in the elevation direction and what elevation angle is assumed for the correction is therefore not crucial (since the correction is only made in the azimuthal direction). Therefore, we assume that the elevation angle is $0^{\circ}$ for sectors $1-10$. For sectors $11-15$, the pixel in the aperture plane is shadowed by the spacecraft body and the elevation angle is increased to 11.25 (elevation bin 10 in Bergman et al. 2020b). If the actual elevation angle used by the instrument is not perfectly centred around the aperture plane, i.e. $0^{\circ}$ elevation, the elevation angle becomes energy dependent. This is not taken into account. These assumptions introduce a small uncertainty, which is analysed in Section 4.1.

\subsubsection{General correction principle}

To correct the RPC-ICA data, we first normalize the flux maps provided by Bergman et al. (2020b) by integrating over the solid angle and dividing by the total flux. We then obtain a flux map with sum 1, which can be considered a probability map. For each pixel, these maps provide the probability that the detected ion originally came from the different directions in the map. The total amount of detected counts for each sector is then multiplied with the corresponding probability map. As already mentioned, we assume that the signal is broad enough to cover the complete FOV. This is done individually for each sector of RPC-ICA, and the final map is obtained by summing the maps for the different sectors. Since we are only correcting in the azimuthal direction, we finally sum over all elevation angles for the new distribution of counts.

We divide the data into energy ranges of 2.5-5, 5-10, 10-20, and $20-40 \mathrm{eV}$, and choose corresponding energy ranges for the simulation results based on the spacecraft potential. For a more accurate interpolation, we extend the simulated energy ranges provided by Bergman et al. (2020b) to 5-10, 7.5-15, 10-20, 15-30, 20-40, 3060 , and $40-80 \mathrm{eV}$. The combination of energies into intervals is necessary due to statistical reasons (a limited amount of particles was used for the particle tracing). Due to the noise level of SPIS, energies below $5 \mathrm{eV}$ were not studied by Bergman et al. (2020b). Lower energies can still be corrected using the scaling procedure described in Section 3.3.2 if the spacecraft potential is less negative than in the previous study, and we include energies down to $2.5 \mathrm{eV}$ for all cases where $U_{\mathrm{s} / \mathrm{c}}>-12.25 \mathrm{~V}$.

In Fig. 4, example data from 2015 November 20, from time 08:46:35 to 08:52:51, are shown before and after correcting the directions. The magnitude of the correction depends on the sectors involved, but is usually around $1-2$ sectors $\left(22^{\circ} .5-45^{\circ}\right)$ for the lowest ion energies and a typical negative spacecraft potential.

\subsubsection{Spacecraft potential estimates}

The method presented in Section 3.2 corrects the energy scale of RPC-ICA for the combined effects of low sensor temperature and spacecraft potential. However, for the direction correction we need an estimate of the spacecraft potential alone, without the effect of 

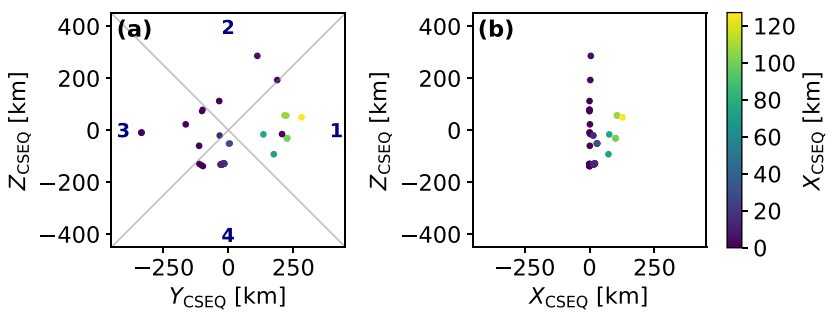

Figure 5. Position of Rosetta in the CSEQ reference frame during the cavity observations used for this study. Panel (a): $Y_{\mathrm{CSEQ}}-Z_{\mathrm{CSEQ}}$ plane. The observations are in this plane divided into the four regions numbered in blue. Panel (b): $X_{\mathrm{CSEQ}}-Z_{\mathrm{CSEQ}}$ plane.

the sensor temperature included. In the band where the potential can be considered constant, the limited time resolution of RPC-LAP is not an issue and we use the closest RPC-LAP estimate for each RPC-ICA data point. In the bursts, this is not sufficient and a high resolution version of the spacecraft potential has to be estimated from the RPC-ICA lower cut-off. For intervals where the sensor temperature is $>13.5 \mathrm{C}$, we estimate the potential using the method described in Section 3.2. For intervals where the sensor temperature is $<13.5 \mathrm{C}$, effects introduced by the low temperature have to be corrected for before the spacecraft potential is determined from the cut-off. This is done by shifting the RPC-ICA spectrum so that the cut-off coincides with the RPC-LAP estimates of the spacecraft potential, where available. We solely use band intervals within each cavity observation for this correction, since the RPC-ICA cut-off is constant in these intervals and more easily determined. For each RPC-ICA data point within the band, we calculate an offset between the cut-off and the closest RPC-LAP data point available. The cavity observations are sufficiently short for the sensor temperature to not change significantly throughout the observation, and to remove the influence of statistical variations, we average all calculated offsets, producing one offset value for each observation. After correcting the spectrum using this method, the high-resolution estimate of the spacecraft potential is obtained as described in Section 3.2.

\section{RESULTS}

In Fig. 5, the position of Rosetta during the diamagnetic cavity observations used for this study is shown in the comet-centred solar equatorial (CSEQ) reference frame. In this frame, the $X_{\mathrm{CSEQ}}$ axis points towards the Sun along the Sun-comet line, the $Z_{\mathrm{CSEQ}}$ axis is orthogonal to $X_{\mathrm{CSEQ}}$ and directed along the solar rotation axis, and the $Y_{\mathrm{CSEQ}}$ axis completes the right-handed system. We divide the observations into four regions according to the clock angle from the $Y_{\mathrm{CSEQ}}$ axis towards the $Z_{\mathrm{CSEQ}}$ axis, in the same way as Masunaga et al. (2019). Region 1 is defined by a clock angle of $-45^{\circ}$ to $+45^{\circ}$, Region 2 by $+45^{\circ}$ to $+135^{\circ}$, Region 3 by $+135^{\circ}$ to $+225^{\circ}$, and Region 4 by $+225^{\circ}$ to $+315^{\circ}$.

We perform a statistical analysis of the flow direction of the ions for all cavity observations, both inside the cavity and just outside. Outside of the cavity, we use data obtained $10 \mathrm{~min}$ before (after) entering (leaving) the cavity. The results for outside and inside the cavity are shown in Figs 6 and 7, respectively. A separation between band and bursts has been done, as explained in Section 3.1. Note that the energy range is different for the band and the bursts. Since the ions in the band predominantly have energies below $20 \mathrm{eV}$, higher energies have not been included in the plot. The ions in the bursts, on the other hand, have slightly higher energies, and energies up to $40 \mathrm{eV}$ have been included.
Since Rosetta was normally orbiting in the terminator plane, sector $7,8,9$, or 10 was usually facing the Sun, while sector 3,4 , or 5 was facing the comet. In this work, the data for each observation have been rotated to align with the direction looking towards the comet. We define a new sector plane with sectors 0 ' $-15^{\prime}$. In this new plane, sector 4' always looks towards the comet. In Figs 6 and 7, the comet and average Sun directions are shown with blue and red arrows, respectively.

Outside the cavity boundary (Fig. 6), the ions in the bursts are predominantly flowing radially outwards from the comet nucleus, with a small antisunward component. Interestingly, the ions in the band are flowing in more or less the opposite direction. The direction of these ions is clearly cometward, contradicting the previous assumptions of a radial anticometward flow of these ions. The flow direction appears to have a small sunward component, but since the original sector 13 is dead, this may be an instrumental effect. On the other hand, sector 0 has been removed from the study, meaning that the sunward component can also be larger than apparent. Precise details about the cometward flow can hence not be obtained from the RPC-ICA data. No apparent differences between the regions are visible.

Inside the cavity (Fig. 7), the statistics are not as good due to a smaller amount of data, especially for regions $1-3$. This makes the exact division between band and bursts important. Since the separation is done manually, the division is generally more exact than for an automatic method, but some parts of the data can still get mischaracterized and, without sufficient statistics, this will affect the result. This is probably the reason for the discrepant features appearing in the band in region 1, and in the bursts in region 3. Apart from these features, the results are similar to the results outside of the cavity boundary. The band ions are flowing predominantly cometward and the burst ions are flowing anticometward with a clear antisunward component. The antisunward component is larger inside the cavity than outside, making the band ions and the burst ions flow in a perpendicular fashion rather than oppositely. Also, in this case, no apparent differences between the regions are visible.

\subsection{Uncertainty analysis}

As discussed in Section 3.3.2, several assumptions have been made to correct the directional information of the data. In this section we analyse the resulting uncertainties introduced by two of these assumptions: the flow direction assumed when modelling the potential field around the spacecraft (affecting the location of the wake) and the elevation angle assumed for the elevation bin used by RPC-ICA in the high-time-resolution mode. To analyse the uncertainty introduced by the flow direction, we run two additional simulations where the flow direction is changed to the $+z$-direction (cometward flow) and the $-x$-direction (antisunward flow) and reprocess the data using these new simulation results. Only one simulation is run for each setup (as compared to five combined simulations for the original, reference, setup), resulting in a larger statistical uncertainty for these results. However, the accuracy should still be sufficient to see systematic, significant differences in the results. For the elevation angle, we change the assumed elevation angle to a more extreme value of 22.5 (elevation bin 12 in Bergman et al. (2020b)) for all sectors and reprocess the data using this new elevation angle. A combination of five simulations was used, as for the reference case. The results are shown in Fig. 8. Since no systematic differences are observed between the regions, we only show the results for region 4 . Also, we only show the results for outside of the cavity, but for both the band and the bursts. No significant differences are observed compared to the reference case, and we conclude that the uncertainties introduced by the different assumptions are, most likely, minor. 

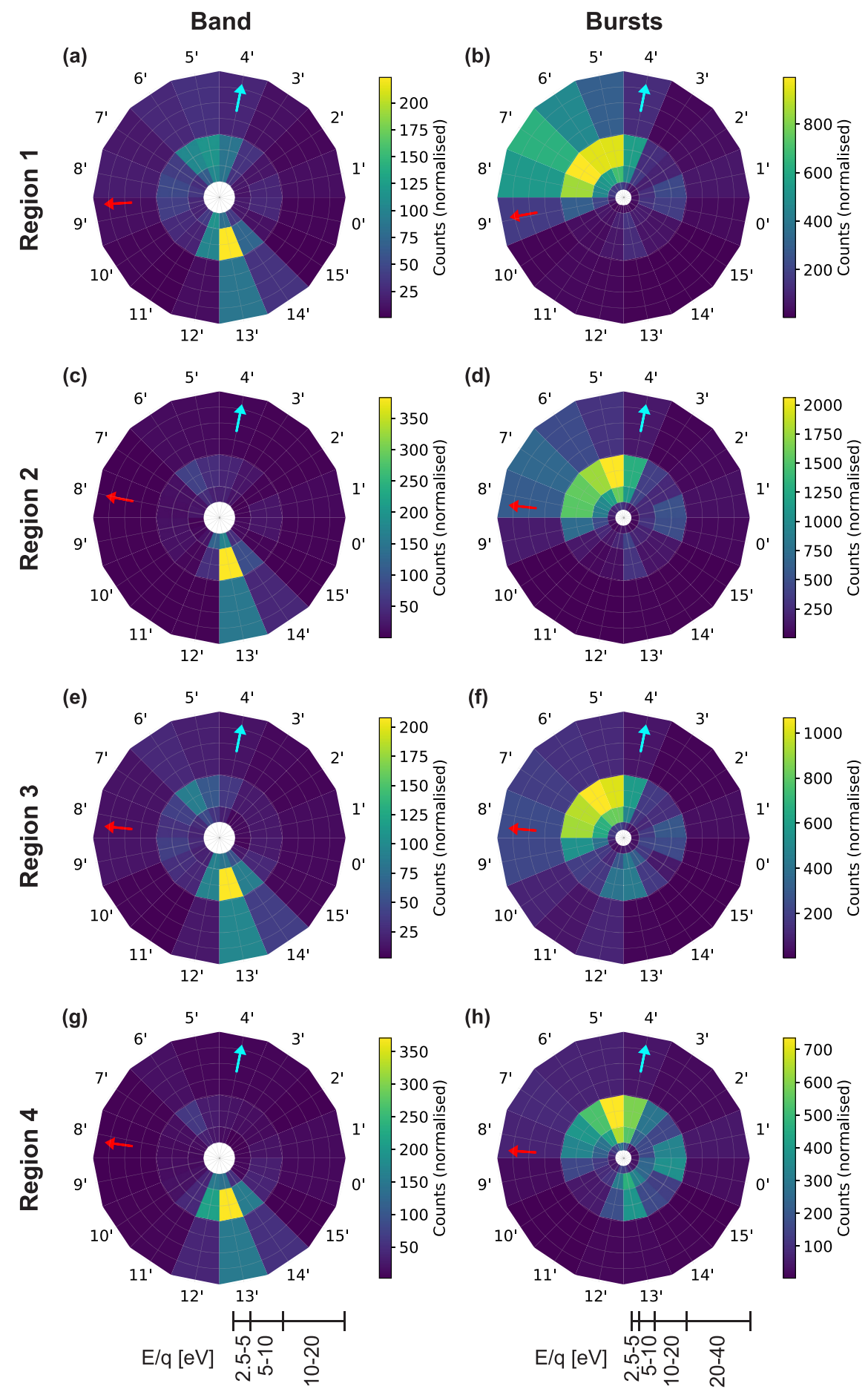

Figure 6. Corrected flow directions of the ions outside of the cavity boundary. In panels (a), (c), (e), and (g), the result for the ions in the band is shown for regions 1, 2, 3, and 4, respectively. In panels (b), (d), (f), and (h), the result for the ions in the bursts is shown. The data have been rotated so that sector 4 ' is always looking cometward, i.e. counts in this sector represent ions moving radially outward from the nucleus. The blue and red arrows show the comet and average Sun directions, respectively. The numbers on the azimuthal axis correspond to the sectors of the new, rotated, sector plane and the radial axis corresponds to energy. Note that the energy range is different for the band and the bursts. The colour scale corresponds to counts detected from different directions, summed over all events. Since measurements of the lowest energy interval is only included when $U_{\mathrm{s} / \mathrm{c}}>-12.25 \mathrm{~V}$, the amount of detected counts for each energy interval has been normalized by the total observation time for that interval. 

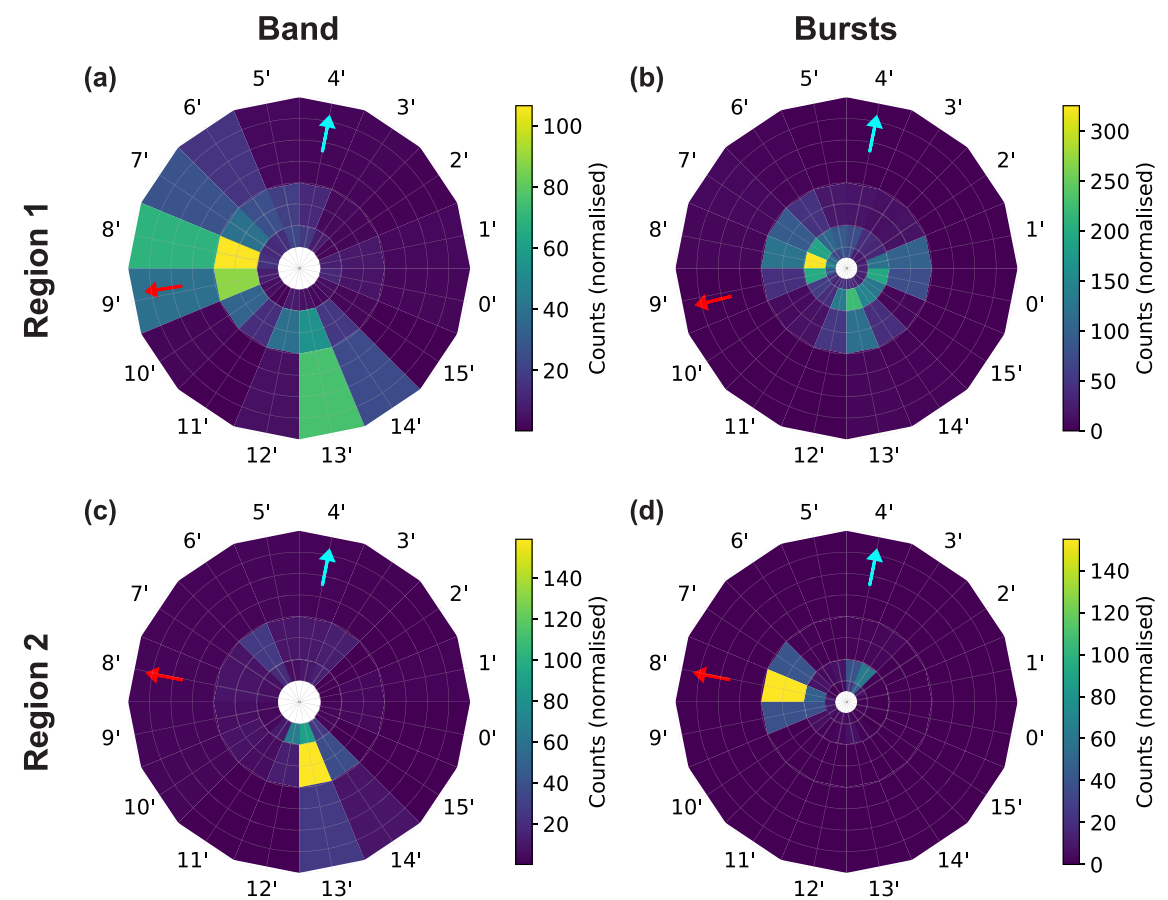

(e)

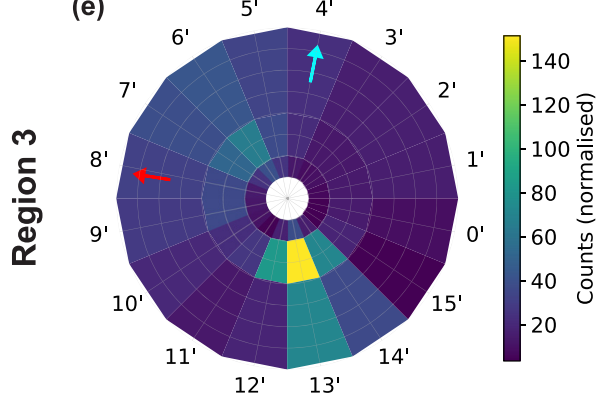

(f)

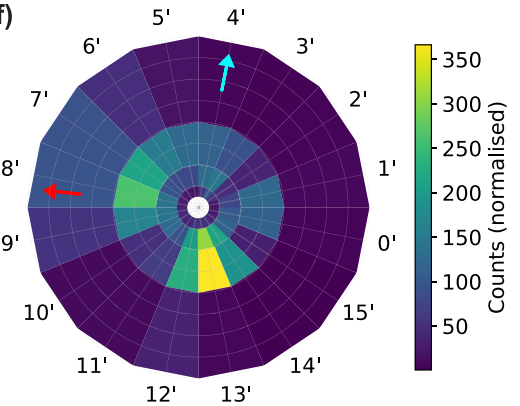

(g)

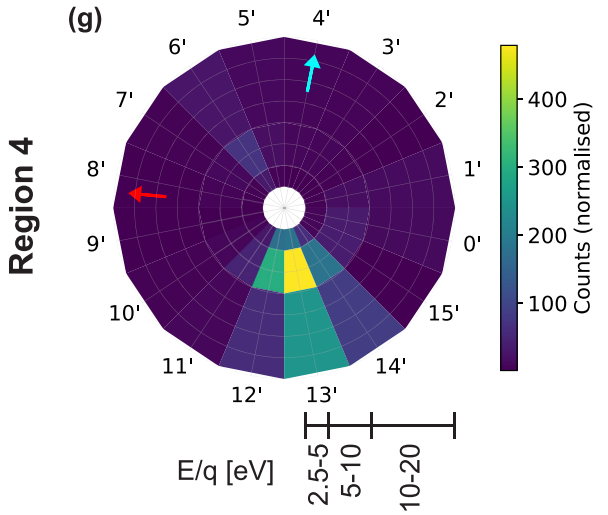

(h)

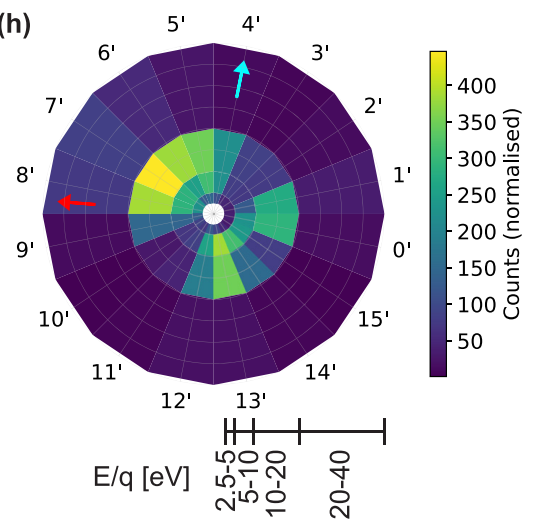

Figure 7. Same as Fig. 6, but for the ions inside the cavity.

\section{DISCUSSION}

\subsection{Band}

One interesting finding is the cometward flow of ions. These ions are moving more or less radially inwards towards the comet nucleus, possibly with a small sunward component. They are present both in the cavity and outside of the cavity boundary, without any apparent difference in flow direction between the two regions. Backstreaming ions connected to the cavity boundary have previously been predicted for comet 1P/Halley by Puhl-Quinn \& Cravens (1995), who did 1D hybrid simulations of the cavity boundary region. These simulations show that ions flowing radially outwards from the nucleus can get magnetically reflected at the boundary and stream back towards the comet again. For comet 67P, the possibility of a reversal of the ambipolar electric field has been discussed by e.g. Vigren \& Eriksson (2019). A time stationary electric field will trap cold electrons that cannot overcome the potential barrier. Unless recombination is highly 

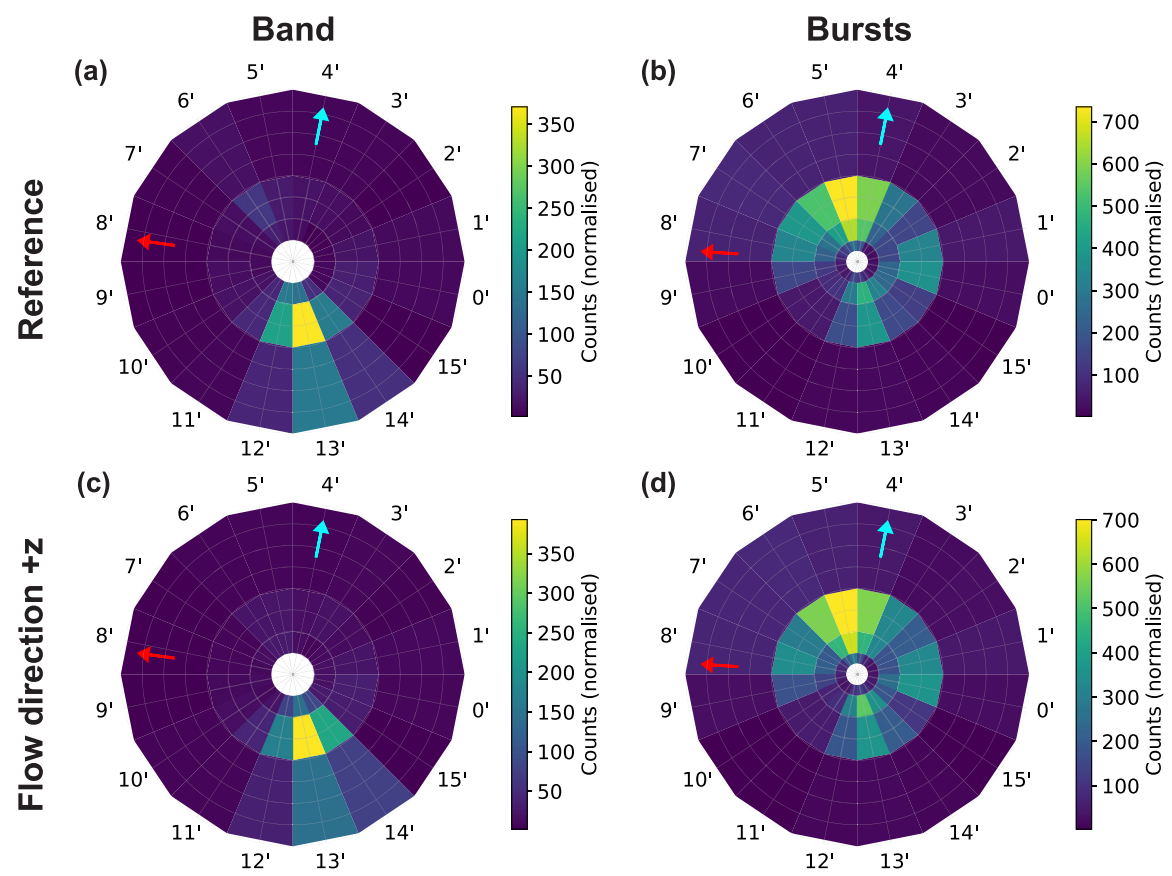

(d)

(e)

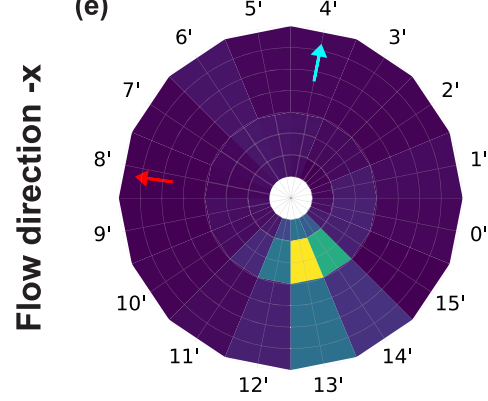

(g)

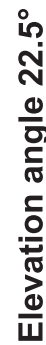
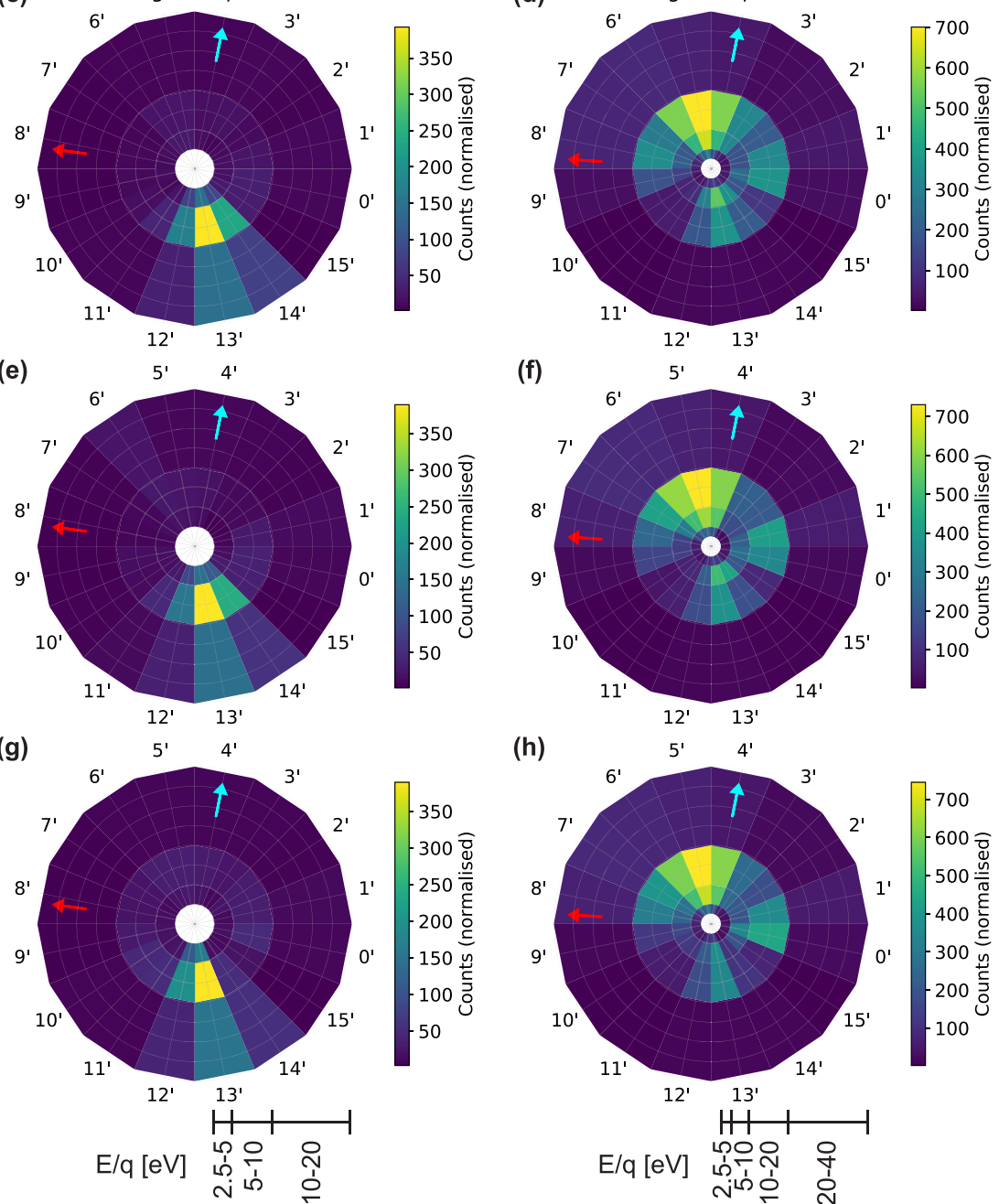

(f)
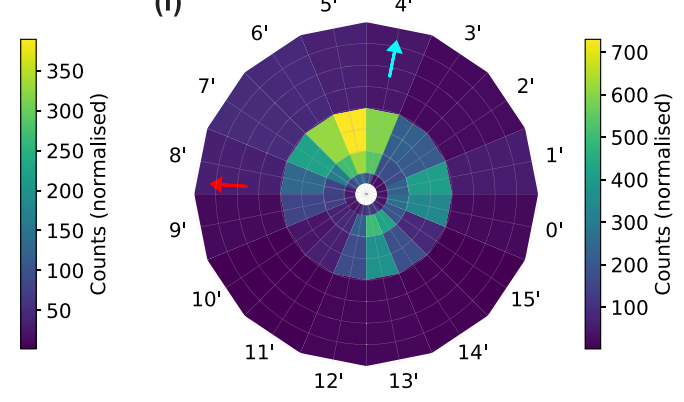

(h)

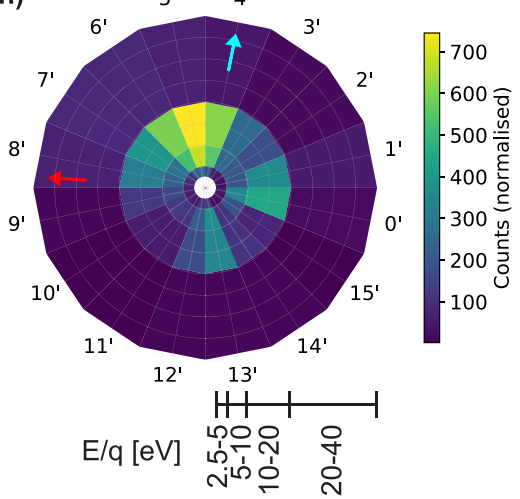

Figure 8. Analysis of the uncertainties introduced by some of the assumptions made during the correction of the data. Only the result for region 4 outside of the cavity is shown. In panels (a)-(b), the reference case is shown (identical to panels g-h in Fig. 6); in panels (c)-(d), the main flow direction for modelling the potential field around the spacecraft has been changed to the $+z$-direction; in panels (e)-(f), the same flow direction has been changed to the $-x$-direction; and in panels $(\mathrm{g})-(\mathrm{h})$, the elevation angle of the assumed elevation bin has been changed to 22.5 (elevation bin 12 in Bergman et al. $2020 \mathrm{~b})$ for all sectors. The format is otherwise the same as in Fig. 6.

efficient, this population will grow until the ambipolar field is reduced or reversed so that they can escape. A time-varying ambipolar field that temporarily can be even reversed is hence not unreasonable.

The main issue with both mechanisms suggested above is the apparent lack of ions flowing away from the nucleus in the data, which would be expected in the presence of an ambipolar electric field. Outside the cavity, the ions in the bursts are flowing more or less radially outwards, but at time periods where only the band is present, no significant anticometward flow is visible. For the bursts inside the cavity, the radial component is very small, maybe even nonexistent, resulting in a constant lack of ions flowing radially outwards in this region. An absence of ions flowing anticometward seems unreasonable, which is supported by modelling results from e.g Koenders et al. (2015), showing that a radial anticometward flow 


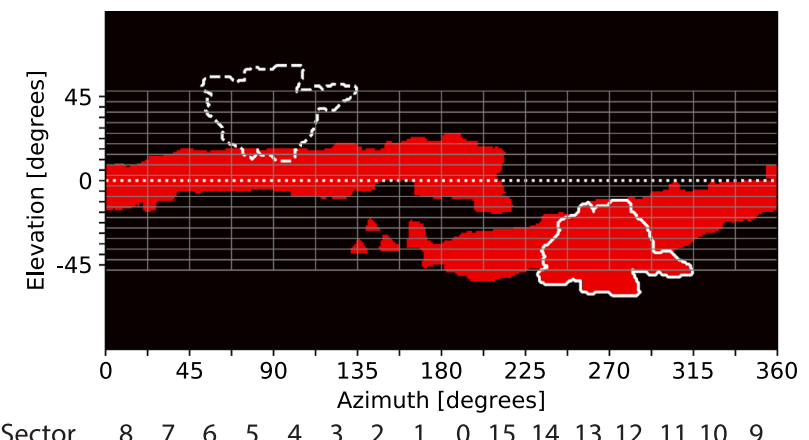

Sector $\quad \begin{array}{llllllllllllllll}8 & 7 & 6 & 5 & 4 & 3 & 2 & 1 & 0 & 15 & 14 & 13 & 12 & 11 & 10 & 9\end{array}$

Figure 9. The effective FOV of RPC-ICA (red area) for a 2D measurement with nominal elevation angle 11.25 when the ion energy at infinity is 5$10 \mathrm{eV}$ and the spacecraft potential is $-21 \mathrm{~V}$. The outer edge of the red area corresponds to the full with at half-maximum of the simulated flux maps. The solid white line encloses the area corresponding to the FOV of sectors 14 and 15 , and the dashed white line shows this FOV mirrored. For reference, the aperture plane has been marked with a dotted white line. The FOV of sectors 0,1 , and 13 have not been included in the figure.

is dominating within the cavity. Measurements made by LAP2 as presented by Odelstad et al. (2018) are also consistent with a flow directed radially outwards from the nucleus. Measurements during spacecraft slews indicate that LAP2 was located in the wake during nominal pointing conditions, which inside the cavity is consistent with a radial anticometward flow. However, since RPC-ICA is measuring in nearly $2 \mathrm{D}$ in the high-time-resolution mode, signals can end up outside of the FOV. Spacecraft potential effects furthermore create an effective FOV that is larger for sectors looking in the $-z$ direction than those looking in the $+z$-direction where the influence of the spacecraft potential generally is smaller. In principle, a signal arriving from the $-z$ hemisphere (a cometward flow) could therefore be more easily detected than one arriving from the opposite direction. This is illustrated in Fig. 9, where the red area is the effective FOV of RPC-ICA for an ion energy of 5-10 eV and a spacecraft potential of $-21 \mathrm{~V}$, assuming a $2 \mathrm{D}$ measurement with an elevation angle of 11.25. The cometward flow is predominantly detected in sectors 14 and 15, with an effective FOV corresponding to the area marked with a white solid line in Fig. 9. The dashed white line is this FOV mirrored. That is, perfectly mirrored ions would have a flow direction somewhere in this region, which is clearly not covered by RPC-ICA at this time. The precise geometry varies with spacecraft potential, ion energy and elevation angle, but this example shows that the diminished FOV of the instrument makes it possible for one signal to remain undetected in the situation of counter-streaming ions. In Fig. 2, a weak anticometward signal is indeed visible in the band-data (see sectors 4, 5, and 6), which may indicate that a counter-streaming signal is located just outside of the FOV of the instrument. However, the comet was for most analysed cavity observations located very close to the aperture plane (a region covered by the FOV in the case shown in Fig. 9), meaning that the flow direction cannot be perfectly radial in the case of a missed signal, but must have a significant $y$-component in the spacecraft frame. However, this depends on the exact elevation angle used by RPC-ICA in the high-time-resolution mode. In case the actual elevation angle is much larger than $0^{\circ}$, the aperture plane is no longer covered by the effective FOV for sectors looking in the $+z$-direction (sectors 3-6), meaning that also a purely radial flow could be missed. Interestingly, if the actual elevation angle is 22.5 , the effective FOV of sectors 14 and 15 covers the aperture plane, which is not the case for sectors looking in the opposite direction, meaning that purely radial bi-directional beams would only be observed in the cometward direction.

To investigate whether the backstreaming ions are connected to processes at the cavity boundary, we look at data from 2016 March, a time period of lower cometary activity where the diamagnetic cavity was not detected. Excluding data from the nightside excursion, RPCICA was running in the high-time-resolution mode during eight time periods of varying length during 2016 March. One example is shown in Figs 10 (uncorrected energy spectrum) and 11 (corrected flow directions). For all data obtained during this month, the sporadic bursts of ions are generally absent, indicating that the origin of these features is connected to the diamagnetic cavity and/or periods of higher outgassing rates (see further discussion in Section 3.2). Ions flowing cometward are still present in the band, indicating that this feature is not connected to the diamagnetic cavity, but more likely the result of a reversal of the ambipolar field or a similar process. However, even though the diamagnetic cavity was not detected by Rosetta in 2016 March, it can still be present closer to the nucleus. If the distance between the spacecraft and the cavity boundary is smaller than the gyroradius of the ions, magnetic reflection can still be responsible for the backstreaming ions. Another prominent feature is that the ions in the band are not only flowing cometward during this time period; a significant part is also flowing radially away from the nucleus, similar to what would be expected, but is not observed, in and around the diamagnetic cavity. This signal is comparable in strength to the anticometward signal. The two flows appear to not be perfectly opposite, even though it is difficult to draw any firm conclusions due to the dead sector 13 of RPC-ICA. The geometry between the spacecraft and the comet as well as the spacecraft potential are similar in 2016 March compared to the cases at the cavity, meaning that if the anticometward signal is present at the cavity as well, but in this case ends up outside of the FOV of RPC-ICA, the elevation angle of the flow has to be different during the cavity cases compared to the 2016 March cases. It is tempting to start speculating about whether the anticometward flux indeed is much lower than the cometward flux during the cavity cases, but this situation would not be easily explained. Another interesting observation is that the model by Vigren et al. (2019), estimating electron number densities assuming that the ion speed is the same as the neutral speed, manage to reproduce the observed densities in 2016 March, but deviates during the rest of the studied period (from 2015 November to 2016 March 21).

Generally, models assuming ions expanding radially outwards with a speed similar to the neutral speed have been able to fairly well reproduce observed densities at large heliocentric distances (e.g. Galand et al. 2016; Vigren et al. 2016, 2019; Heritier et al. 2018). Observations of the ion speed, on the other hand, indicate speeds significantly above the speed of the neutral particles (e.g. Odelstad et al. 2018; Bergman et al. 2021). The presence of counter-streaming ions could be able to explain this discrepancy, since even though individual parts of the population have bulk speeds significantly above the speed of the neutrals, the net expansion velocity can be lower.

Counter-streaming ions can furthermore be a source of wave generation, which can explain the observations of ion acoustic waves inside the cavity (Gunell et al. 2017).

\subsection{Bursts}

The bursts are, except for their flow direction, well connected to the band ions in terms of the lower energies. After correcting for the spacecraft potential, the whole spectrum more or less appears as a 
Sector 0
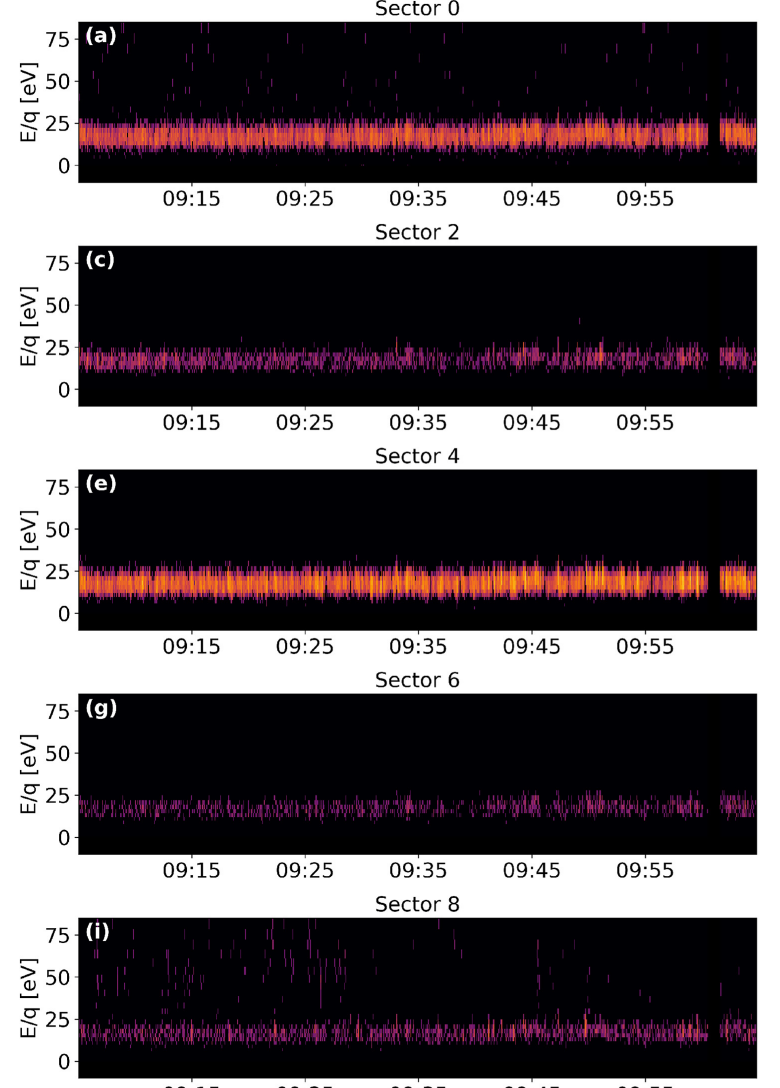

09:15
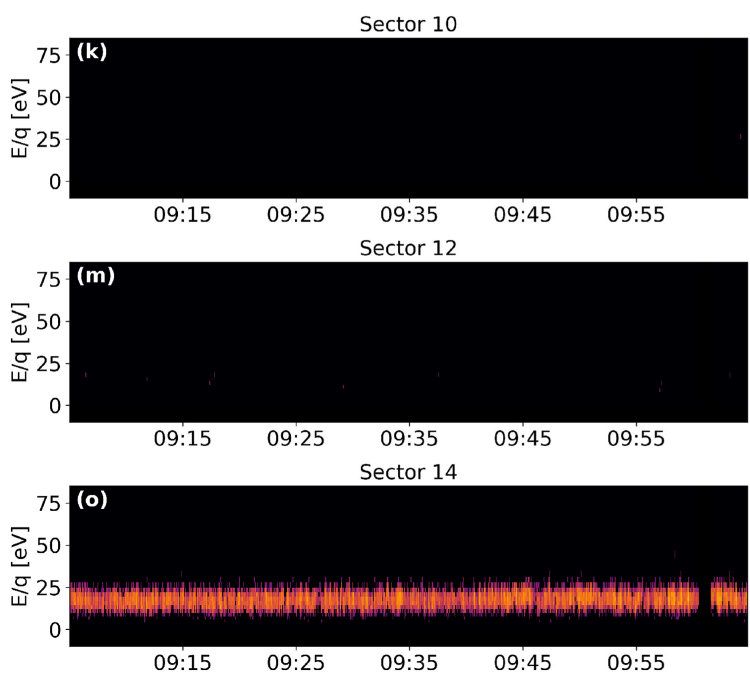
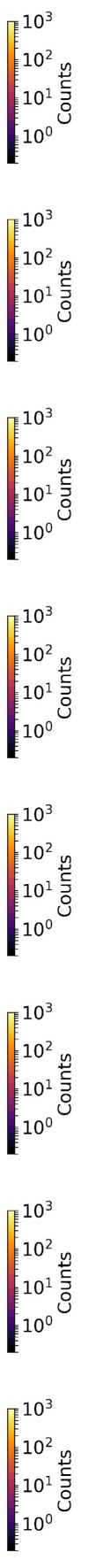

Sector 1
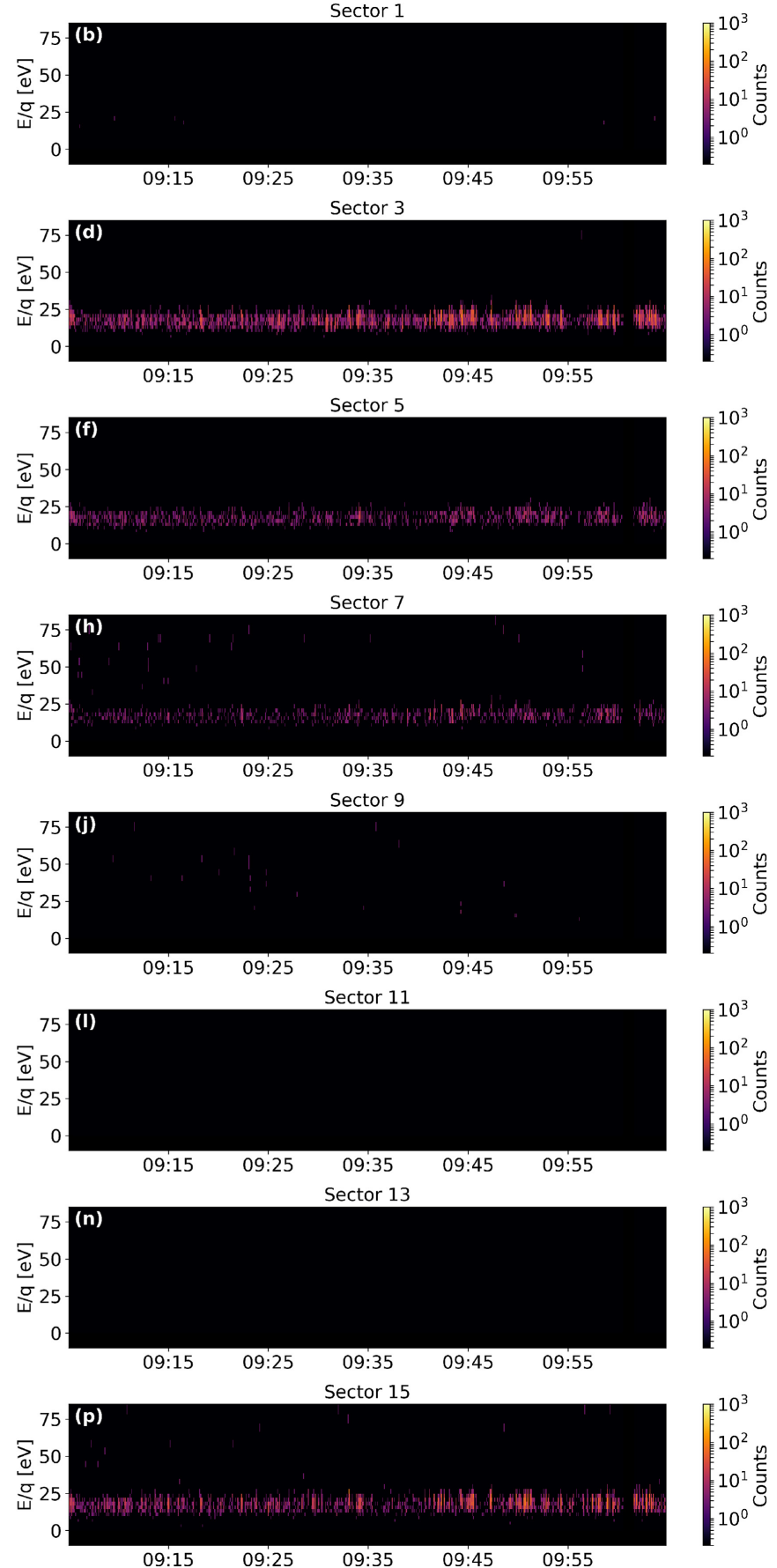

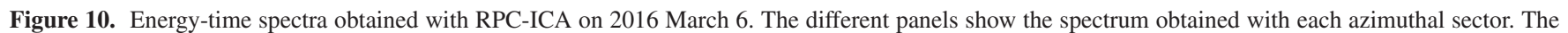
data have not been corrected for the spacecraft potential.

constant band with spikes of accelerated ions appearing in the burst features. Hence, we have no reason to believe that these are two different ion populations with different origin. Rather, we propose that the bursts are created from the band due to some compressional mechanism, perhaps connected to the cavity boundary. The issue with this hypothesis is that the bursts are seemingly appearing from out of nowhere; again we would expect the band ions to be also present in the direction of the bursts. However, if the band flow in this direction is outside of the FOV of RPC-ICA, as was previously discussed, it would be possible for the bursts to show in the data if they are accompanied by, for example, a temperature increase. The spacecraft potential furthermore becomes more negative in the bursts, which, in principle, can make an otherwise invisible signal detectable in those instances. However, because of reason (ii) discussed in the next paragraph, the spacecraft potential is most likely not the cause in this case. The flow direction outside the cavity is similar to the propagation direction of the steepened waves analysed by Ostaszewski et al. (2021), confirming the compressional nature of these waves. No separation has been made between steepened features and other observed burst features since this is outside the 
scope of this study. However, there are no indications that the flow direction of the different features differ significantly. Inside the cavity, the flow direction has a larger antisunward component. As already concluded by Hajra et al. (2018), the bursts in the cavity are most likely generated at, or transmitted from outside of, the cavity boundary. It was shown in the study by Hajra et al. (2018) that the plasma enhancements are not accompanied by a change in the neutral density and, since photoionization is expected to dominate close to perihelion, this makes it difficult to find a mechanism that can generate these features within the cavity. The diffusion timescales were furthermore shown by the same study to be too short for plasma structures generated in the inner coma to be observable by Rosetta. The transmission mechanism and the coupling between these features and the magnetic field is a topic for a future study.

\subsection{Final remarks}

Finally, we want to make some remarks about the possibility of the different flow directions observed for the ions in the bursts and the band to be an effect of the spacecraft potential. In the bursts, the spacecraft potential becomes more negative, which causes a larger distortion of the effective FOV. However, we find it highly unlikely that the spacecraft potential is responsible for the difference observed in this study, due to mainly four reasons: (i) According to the simulations by Bergman et al. (2020b), a spacecraft potential variation of this magnitude cannot cause a change in observed flow direction of several sectors. (ii) No other correlation with the spacecraft potential is observed. The spacecraft potential in the constant part of the band varies between different cavity observations; in some cases the spacecraft potential in this part of the band is more negative than the spacecraft potential when the bursts appear for another observation. The difference in flow direction between the band and bursts is still the same. (iii) In 2016 March, ions are observed from both directions at the same time. (iv) When the bursts appear, the band is generally still present, with ions arriving from the same direction as before the arrival of the burst.

Hence, we conclude that the different flow directions observed for the band and the bursts are most likely real, and not an effect of the spacecraft potential.

\section{CONCLUSIONS}

In this study, we have constrained the flow directions of lowenergy ions in and around the diamagnetic cavity of comet 67P. We used high-time-resolution data from RPC-ICA obtained during the time period from 2015 May to 2016 February, corrected for the spacecraft potential using the PIC simulation results from Bergman et al. (2020b). A separation of the data was made between slightly accelerated 'burst' features and a more constant 'band' of low-energy ions. The results show the following:

(i) The ions in the band are flowing predominantly cometward, providing evidence for the existence of counter-streaming ions, as previously proposed by e.g. Puhl-Quinn \& Cravens (1995) and Vigren \& Eriksson (2019). The backstreaming ions are observed both inside and outside of the cavity, and also during times of lower cometary activity where a diamagnetic cavity was not detected, indicating that the process responsible for accelerating these ions cometward is not connected to the cavity boundary.

(ii) The amount of ions flowing radially outwards from the nucleus in the RPC-ICA data is surprisingly small, indicating that the flow direction of these ions is outside of the FOV of the instrument.

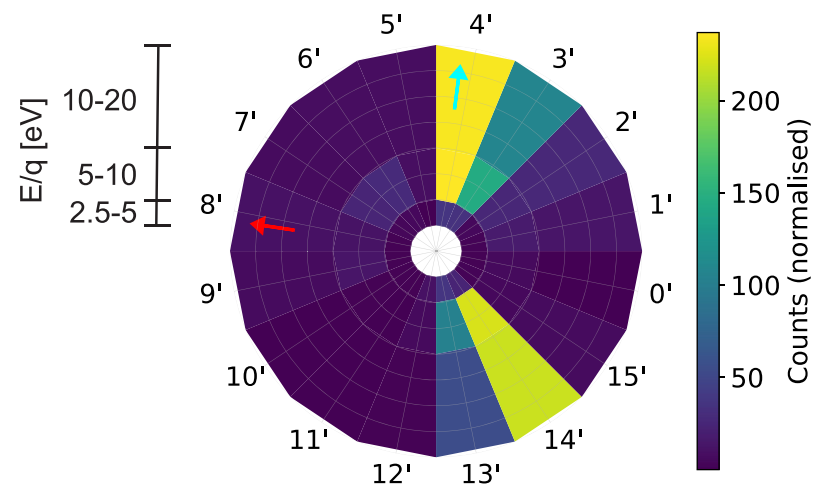

Figure 11. Corrected flow directions for the time period on 2016 March 6 shown in Fig. 10. On the azimuthal axis, the new sector numbers 0'-15' are shown and the radial axis corresponds to energy. The unit of the colour scale is counts, normalized by the observation time for each energy interval.

(iii) In 2016 March, a time period when the diamagnetic cavity was not detected, counter-streaming ions are clearly visible in the data with a cometward and an anticometward component of comparable strength.

(iv) Outside of the cavity, the ions in the bursts are flowing radially outwards from the nucleus with a small antisunward component. Inside the cavity, the flow of the bursts still has a small anticometward component but is predominantly antisunward. The bursts are well connected to the ions in the band in terms of the lower energies (the whole spectrum more or less appears as a constant band after correcting for the spacecraft potential), and we propose a creation mechanism where the bursts are created through compression of the ions in the band, perhaps at the cavity boundary. The burst features observed in the cavity are most likely generated at, or transmitted from outside of, the cavity boundary.

(v) The bursts are seemingly appearing from 'out of nowhere', indicating that the flow from which they are created are located outside of the FOV of the instrument. The sudden appearances of the bursts do not seem to be connected to the variations of the spacecraft potential, but could be a result of a temperature increase in the bursts.

\section{ACKNOWLEDGEMENTS}

The work of S. Bergman and G. Stenberg Wieser is supported by the Swedish National Space Agency (SNSA) through grants 130/16 and 96/15, respectively. The work of Arnaud Beth at Umeå University is supported by the Swedish National Space Agency, grant 108/18. Rosetta is a European Space Agency (ESA) mission with contributions from its member states and the National Aeronautics and Space Administration (NASA). The RPC-ICA and RPC-LAP instruments are supported by SNSA. We acknowledge the Spacecraft Plasma Interaction Network In Europe (SPINE) for the development of the SPIS software.

\section{DATA AVAILABILITY}

The data underlying this paper have been submitted to the ESA Planetary Science Archive and will be available through https://psa. esa.int. At the time of submission, the data have been approved but are not yet ingested into the PSA data base. The SPIS software is freely available at https://www.spis.org/software/spis/. 


\section{REFERENCES}

Alfvén H., 1957, Tellus, 9, 92

Auster H.-U. et al., 2015, Science, 349, aaa5102

Behar E., Lindkvist J., Nilsson H., Holmström M., Stenberg-Wieser G., Ramstad R., Götz C., 2016, A\&A, 596, A42

Behar E., Tabone B., Saillenfest M., Henri P., Deca J., Lindkvist J., Holmström M., Nilsson H., 2018, A\&A, 620, A35

Berčič L., Behar E., Nilsson H., Nicolaou G., Stenberg Wieser G., Wieser M., Goetz C., 2018, A\&A, 613, A57

Bergman S., Stenberg Wieser G., Wieser M., Johansson F. L., Eriksson A., 2020a, J. Geophys. Res.: Space Phys., 125, e2020JA027870

Bergman S., Stenberg Wieser G., Wieser M., Johansson F. L., Eriksson A., 2020b, J. Geophys. Res.: Space Phys., 125, e2019JA027478

Bergman S. et al., 2021, MNRAS, 503, 2733

Cravens T. E., 1989, J. Geophys. Res.: Space Phys., 94, 15025

Eriksson A. I. et al., 2007, Space Sci. Rev., 128, 729

Eriksson A. I. et al., 2017, A\&A, 605, A15

Galand M. et al., 2016, MNRAS, 462, S331

Gilet N. et al., 2020, A\&A, 640, A110

Glassmeier K.-H., Boehnhardt H., Koschny D., Kührt E., Richter I., 2007a, Space Sci. Rev., 128, 1

Glassmeier K.-H. et al., 2007b, Space Sci. Rev., 128, 649

Goetz C. et al., 2016a, MNRAS, 462, S459

Goetz C. et al., 2016b, A\&A, 588, A24

Gulkis S. et al., 2015, Science, 347, aaa0709

Gunell H. et al., 2017, MNRAS, 469, S84

Hajra R. et al., 2018, MNRAS, 475, 4140

Henri P. et al., 2017, MNRAS, 469, S372

Heritier K. L. et al., 2018, A\&A, 618, A77

Ip W., Axford W., 1987, Nature, 325, 418
Johansson F. L., Eriksson A. I., Gilet N., Henri P., Wattieaux G., Taylor M. G. G. T., Imhof C., Cipriani F., 2020, A\&A, 642, A43

Johansson F. L. et al., 2021, A\&A, in press

Koenders C., Glassmeier K.-H., Richter I., Ranocha H., Motschmann U., 2015, Planet. Space Science, 105, 101

Masunaga K., Nilsson H., Behar E., Stenberg Wieser G., Wieser M., Goetz C., 2019, A\&A, 630, A43

Neubauer F. M. et al., 1986, Nature, 321, 352

Nilsson H. et al., 2007, Space Sci. Rev., 128, 671

Nilsson H. et al., 2018, A\&A, 616, A50

Nilsson H. et al., 2020, MNRAS, 498, 5263

Odelstad E., Stenberg-Wieser G., Wieser M., Eriksson A. I., Nilsson H., Johansson F. L., 2017, MNRAS, 469, S568

Odelstad E. et al., 2018, J. Geophys. Res.: Space Phys., 123, 5870

Ostaszewski K. et al., 2021, Ann. Geophys., 39, 721

Puhl-Quinn P., Cravens T. E., 1995, J. Geophys. Res.: Space Phys., 100, 21631

Schwenn R., Ip W. H., Rosenbauer H., Balsiger H., Buhler F., Goldstein R., Meier A., Shelley E. G., 1987, A\&A, 187, 160

Simon Wedlund C. et al., 2017, A\&A, 604, A73

Stenberg Wieser G. et al., 2017, MNRAS, 469, S522

Szegö K. et al., 2000, Space Sci. Rev., 94, 429

Thiébault B., Mateo-Velez J.-C., Forest J., Sarrailh P., 2013, SPIS 5.1 User Manual, ONERA and Artenum, Paris

Trotignon J. G. et al., 2007, Space Sci. Rev., 128, 713

Vigren E., Eriksson A. I., 2017, AJ, 153, 150

Vigren E., Eriksson A. I., 2019, MNRAS, 482, 1937

Vigren E. et al., 2016, AJ, 152, 59

Vigren E. et al., 2017, MNRAS, 469, S142

Vigren E. et al., 2019, ApJ, 881, 6

Wattieaux G., Henri P., Gilet N., Vallières X., Deca J., 2020, A\&A, 638, A124

\section{APPENDIX A: CAVITY OBSERVATIONS USED FOR THE STUDY}

In Table A1, the cavity observations used for this study are listed.

Table A1. Cavity observations used for the study.

\begin{tabular}{lccc}
\hline Day & No. of observations & Total time in cavity $(\mathrm{h} \mathrm{ms})$ & Spacecraft position (region) \\
\hline $2015-05-25$ & 1 & $0: 02: 27$ & 1 \\
$2015-05-26$ & 1 & $0: 00: 47$ & 1 \\
$2015-05-27$ & 1 & $0: 02: 37$ & 1 \\
$2015-05-29$ & 2 & $0: 04: 34$ & 1 \\
$2015-05-30$ & 1 & $0: 02: 41$ & 1 \\
$2015-07-15$ & 1 & $0: 07: 52$ & 3 \\
$2015-07-26$ & 27 & $1: 11: 37$ & 4 \\
$2015-08-02$ & 1 & $0: 01: 05$ & 1 \\
$2015-08-06$ & 1 & $0: 06: 17$ & 2 \\
$2015-08-09$ & 1 & $0: 01: 43$ & 2 \\
$2015-08-21$ & 2 & $0: 03: 26$ & 3 \\
$2015-11-15$ & 1 & $0: 02: 23$ & 4 \\
$2015-11-20$ & 23 & $4: 05: 13$ & 3 \\
$2015-11-23$ & 1 & $0: 04: 42$ & 3 \\
$2015-11-27$ & 3 & $0: 02: 56$ & 2 \\
$2015-11-29$ & 1 & $0: 02: 18$ & 4 \\
$2016-01-31$ & 5 & $0: 08: 26$ & 3 \\
$2016-02-17$ & 2 & $0: 01: 19$ & \\
\hline
\end{tabular}

This paper has been typeset from a $\mathrm{T}_{\mathrm{E}} \mathrm{X} / \mathrm{LT} \mathrm{E} \mathrm{X}$ file prepared by the author. 